\title{
La importancia de introducir la competencia intercultural en la educación superior: Propuesta de actividades prácticas
}

\author{
The importance of Introducing Intercultural Competence in Higher Education: \\ Proposal of Practical Activities
}

\section{A importância da introdução de competência intercultural no ensino superior: Proposta de atividades práticas}

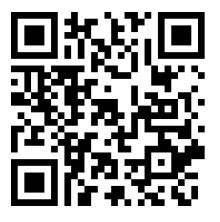

\author{
María Martínez-Lirola \\ Universidad de Alicante \\ Alicante, España \\ maria.lirola@ua.es
}

http://orcid.org/0000-0002-6427-425X

Recibido $\cdot$ Received $\cdot$ Recebido: 15 / 07 / 2016
Corregido $\cdot$ Revised $\cdot$ Revisado: 31 / 05 / 2017
Aceptado $\cdot$ Accepted $\cdot$ Aprovado: 17/ 07 / 2017

Resumen: Enseñar una lengua extranjera como el inglés lleva consigo no solo enseñar contenidos relacionados con la lengua objeto de estudio, sino también introducir aspectos culturales que ayuden a aprender la lengua enmarcada en la cultura. Este artículo es una contribución de cómo introducir aspectos culturales en el aula de lengua extranjera en la educación superior. Se diseñaron distintas actividades cooperativas que favorecían la adquisición de la competencia intercultural, y

${ }^{1}$ Doctora en Filología Inglesa y profesora titular de Filología Inglesa de la Universidad de Alicante. Además, es Research Fellow del Departamento de Lingüística y Lenguas Modernas de la Universidad de Sudáfrica (UNISA). Ha dirigido varios proyectos de investigación sobre análisis crítico del discurso e innovación educativa financiados por entidades públicas. Sus principales líneas de investigación son el análisis crítico del discurso, la gramática sistémica funcional y la lingüística aplicada. Ha publicado alrededor de un centenar de textos que incluyen libros, artículos en revistas nacionales e internacionales, capítulos de libro, ponencias en congresos, reseñas y trabajos de divulgación, además de haber creado y dirigido el grupo de investigación "Análisis Crítico del Discurso Multimodal". En el 2010 obtuvo el Premio a la Excelencia Docente de la Generalitat Valenciana. Ha participado en congresos e impartido conferencias y seminarios en España y en decenas de países. Por otro lado, ha llevado a cabo estancias de investigación con el fin de llevar a cabo investigaciones de temática social y de didáctica. 
doi: http://dx.doi.org/10.15359/ree.22-1.3

URL: http://www.una.ac.cr/educare

CORREO: educare@una.cr

se optó por una metodología cooperativa para facilitar que el alumnado asumiera un papel activo y adquiriera competencias sociales. Además, se preparó una encuesta con el fin de conocer su opinión sobre distintos aspectos relacionados con la competencia intercultural. El alumnado participante en el estudio cursaba la asignatura obligatoria Lengua Inglesa $V$ en el tercer curso del grado en Estudios Ingleses. Se presentan los resultados por ser significativos, debido a la importancia que el alumnado le concede a esta competencia y a cómo valora positivamente trabajar con ella en las actividades propuestas. Se concluye que es fundamental introducir esta competencia en el siglo XXI, con el fin de que el alumnado crezca como ciudadanía global y la docencia esté unida a las demandas sociales.

Palabras claves: Aprendizaje cooperativo; competencia intercultural; educación superior; proceso de enseñanza-aprendizaje.

Abstract: Teaching a foreign language like English involves teaching aspects, not only related to the target language content, but also the introduction of cultural aspects that help to learn the language embedded in a culture. This article is a contribution that shows how to include cultural aspects in the foreign language classroom in higher education. Different cooperative activities favoring the acquisition of intercultural competence were designed, and a cooperative methodology was chosen to make it easier for students to have an active role and acquire social competences. In addition, a survey was prepared to know the opinion of students on different aspects of intercultural competence. The students participating in the study were enrolled in the English Language V mandatory course, in the third year of the degree in English Studies. The results presented are significant, thanks to the importance students attach to this competence, and to how they appreciate working with it in the proposed activities. It is concluded that it is essential to introduce this competence in the twenty-first century, so that students grow as global citizens and teaching is linked to social demands.

Keywords: Cooperative learning; higher education; intercultural competence; teaching-learning process.

Resumo: Ensinar uma língua estrangeira como o Inglês envolve ensinar não apenas aspectos relacionados com o conteúdo da língua, mas também os aspectos culturais que ajudam a aprender a língua enraizada dentro de uma cultura. Este artigo é uma contribuição ao modelo de como introduzir aspectos culturais na aula de língua estrangeira no ensino superior. A proposta foi apresentar diferentes atividades de cooperação que ajudariam na aquisição de competências interculturais, e optou-se por uma metodologia cooperativa para capacitar os alunos a assumir um papel ativo e adquirir habilidades sociais. Além disso, foi preparada uma pesquisa para saber as opiniões sobre diferentes aspectos da competência intercultural. Os estudantes que participaram da pesquisa faziam a disciplina obrigatória de Inglês $V$ no terceiro ano da licenciatura em Estudos Ingleses. Os resultados são apresentados como significativos por causa da importância dada pelos estudantes a esta competência e também como positivos por ser trabalhado com eles nas atividades propostas. Conclui-se que é fundamental introduzir esta competência no século XXI, para que os alunos cresçam em cidadania global e o ensino esteja relacionado com as demandas sociais.

Palavras-chave: aprendizagem cooperativa; competência intercultural; educação superior; ensinoaprendizagem. 


\section{Introducción}

Nuestra sociedad se caracteriza por su carácter global, pues gracias a las nuevas tecnologías es posible estar conectado y conocer la realidad social de otros países en principio alejados del nuestro (Francisco, 2010). De hecho, los flujos migratorios han cambiado el paisaje humano, por lo que es necesario reflexionar sobre la interculturalidad en la sociedad actual (Priegue y Leiva, 2012). Si nos centramos en la educación superior, en concreto en el grado en estudios ingleses, profundizar en otras culturas es importante, pues permite al alumnado estar interconectado con la realidad cultural de la lengua extranjera que estudia, además de ayudarle a comunicarse y poder trabajar en contextos internacionales donde es necesario valorar estos aspectos (Vinagre, 2014). Esto lleva consigo que el alumnado avance en su construcción como sujeto de ciudadanía global, al ser consciente de lo que ocurre en otros lugares y de las relaciones entre situaciones que en principio parecen desligadas de su contexto inmediato y con su propia realidad.

Una de las características fundamentales de la educación superior en el siglo XXI ha de ser la de basar la enseñanza en competencias que sirvan para adaptarse a las demandas de la sociedad. Siguiendo a Lasnier (2000), la competencia consiste en saber hacer tras integrar, movilizar y adecuar tanto conocimientos como capacidades y habilidades. Dichas competencias han de incluir también las sociales, con el fin de contribuir al desarrollo integral del alumnado (Bisquerra, 2008; Bisquerra y Pérez, 2007), de modo que se vea una aplicación directa entre lo que se aprende en las aulas y el mercado laboral (Farh, Seo y Tesluk, 2012).

Nos parece fundamental que, aparte de trabajar la competencia lingüística en las clases de lengua inglesa, se profundice en competencias sociales e interculturales que ayuden a tomar conciencia de la importancia de la interculturalidad al alumnado de lenguas extranjeras (Beck, Kumschick, Eid, y Klann-Delius, 2011). De acuerdo con Bisquerra y Pérez (2007, p. 72), entendemos la competencia social como"... la capacidad para mantener buenas relaciones con otras personas. Esto implica dominar las habilidades sociales, capacidad para la comunicación efectiva, respeto, actitudes pro-sociales, asertividad, etc.". Estas competencias son importantes tanto para las relaciones que se establecen dentro del aula, como para las que se concretan con las personas de otras culturas por medio del análisis de los textos.

Tallon y Sikora (2011) dividen las competencias sociales en dos tipos: a) las que tienen que ver con la sintonía con las demás personas (incluye empatía, conciencia política, comunicación) y b) las que tienen que ver con la construcción de relaciones (incluye cooperación, liderazgo, influencia y gestión de conflictos).

En este sentido, este artículo pretende ser una contribución a cómo el alumnado desarrolla su conciencia crítica, adquiere competencias sociales y se convierte en más intercultural por medio de actividades de temática social en clases de lengua inglesa. Por lo tanto, esta investigación pretende contribuir a señalar la importancia de la adquisición de distintas competencias, entre las 
doi: http://dx.doi.org/10.15359/ree.22-1.3

URL: http://www.una.ac.cr/educare

CORREO: educare@una.cr

que destaquen las sociales e interculturales, no solo para el enriquecimiento individual y la mejora de la capacidad comunicativa en una lengua extranjera, sino también para que el alumnado desarrolle capacidades que le permitan colaborar y relacionarse con personas de otras culturas.

Optaremos por una metodología cooperativa con el fin de que el alumnado aprenda con otras personas y pueda profundizar en las competencias sociales anteriormente mencionadas; a la vez que profundiza en aspectos interculturales, al llevar a cabo distintas actividades con el uso de textos reales. Siguiendo a Priegue y Leiva (2012, p. 2): "Del mismo modo, para materializar los conceptos de interactividad, interconexión, instantaneidad, intercambio, conocimiento, enriquecimiento y ciudadanía intercultural, es necesaria una estrategia metodológica basada en el aprendizaje cooperativo, participativo ...." En el aprendizaje cooperativo el diálogo es la base del aprendizaje, de modo que en una metodología cooperativa se posibilita fomentar un buen clima en el aula por medio de interacciones en las que se respeten las distintas opiniones (D. W. Johnson y F. P. Johnson 2009; D. W. Johnson y R. T. Johnson, 2009; Prieto, 2007).

Las principales diferencias entre el aprendizaje cooperativo y el tradicional en pequeños grupos es que se concede importancia tanto a la tarea como al proceso, además se potencia la heterogeneidad, el liderazgo compartido, la interdependencia positiva del alumnado, a la vez que la responsabilidad individual, mientras se trabaja junto con el fin de obtener un objetivo común. También es importante la adquisición de habilidades sociales y a la reflexión del trabajo grupal.

Por el contrario, en los grupos tradicionales, por un lado, se concedía importancia solo al proceso, primaba la homogeneidad y el liderazgo individual; por otrolado, ni la interdependencia positiva ni la responsabilidad individual jugaban un papel importante. Al trabajar por grupos tradicionales, se asume que el alumnado posee habilidades sociales y no se suele conceder importancia a la autorreflexión (D. W. Johnson y F. P. Johnson, 2009; Prieto, 2007).

El objetivo principal de esta investigación es introducir el componente cultural a través de la propuesta de actividades que se presenta, como, por ejemplo, trabajar con textos multimodales de temática social (sobre diferentes etnias, género) durante el proceso de enseñanza-aprendizaje. Con las actividades de la propuesta presentada más adelante, se introducen aspectos interculturales en la clase de inglés, de modo que se contribuye a que el alumnado sea más intercultural y adquiera competencias sociales a la vez que aprende la lengua extranjera. Además, se diseñó una encuesta con el fin de hacer reflexionar al alumnado sobre la competencia intercultural y conocer su opinión sobre algunos aspectos relacionados con la interculturalidad (Apéndice A).

Las principales preguntas que nos planteamos en esta investigación son: ¿Qué tipo de actividades pueden contribuir a que el alumnado sea más intercultural y a introducir contenidos sociales en el proceso de enseñanza-aprendizaje? ¿Qué opina el alumnado sobre determinados aspectos relacionados con la interculturalidad? 
Basar la asignatura en contenidos sociales y culturales facilita el desarrollo de la conciencia social del alumnado y su responsabilidad, debido a que se potencia que tome conciencia de las distintas realidades, entre las que puede haber situaciones de desigualdad o injusticia social. En consecuencia, el alumnado avanza en el proceso de ser ciudadanía activa, al ampliar su perspectiva conociendo realidades sociales distintas de la propia, tal y como se podrá ver en las actividades propuestas más adelante.

\section{Estado de la cuestión}

Las sociedades actuales son cada vez más interculturales, de modo que las personas en general y el alumnado universitario en particular necesitan formarse con el fin de saber desenvolverse satisfactoriamente en un entorno cada vez más diverso e intercultural. Es decir, convivir, relacionarse y comunicarse con personas de diferentes culturas requiere ciertas competencias entre las que destacan las sociales y las interculturales. En general el profesorado tiene poca formación cultural para introducir el componente cultural en las aulas (Priegue y Leiva, 2012).

Partimos de la idea de que todo aprendizaje tiene su origen en un entorno social. Sin embargo, además del entorno propio, es necesario introducir aspectos de otros entornos, especialmente en el caso de la lengua inglesa, por tratarse de una lengua franca extendida por todo el mundo, de modo que el alumnado pueda conocer aspectos de otras culturas y adquirir competencias interculturales, o "the ability to understand and relate to people from other countries" [La capacidad de entender y relacionarse con personas de otros países] (Byram 1997, p. 5). Byram (1997) señala que es más importante establecer y mantener relaciones con otras personas que el intercambio de información en sí. Así, Byram (1997) afirma que ser competente desde el punto de vista intercultural implica ser capaz de interactuar de modo efectivo, usando recursos lingüísticos y no lingüísticos, con personas de otro país en una lengua extranjera, definición que adoptamos en este artículo.

Desde los años noventa, las competencias interculturales han sido objeto de aprendizaje en las lenguas extranjeras (Byram, 1997, 2000; Byram y Fleming, 1998; Meyer, 1991), y aparecen definiciones de lo que implica ser competente desde el punto de vista intercultural, como por ejemplo: "to behave adequately ... in a flexible manner when confronted with actions, attitudes and expectations of representatives of foreign cultures" [Comportarse adecuadamente ... de una manera flexible frente a acciones, actitudes y expectativas de representantes de culturas extranjeras] (Meyer, 1991, p. 138). Siguiendo a Clouet (2013), las competencias interculturales pueden definirse como la habilidad estudiantil para relacionar su propia cultura con la cultura extranjera, la habilidad para superar relaciones estereotipadas, la habilidad para ser sensibles y usar una variedad de estrategias para contactar con personas de otras culturas y la capacidad para tratar, de forma efectiva, los malos entendidos interculturales y las situaciones conflictivas. 
doi: http://dx.doi.org/10.15359/ree.22-1.3

URL: http://www.una.ac.cr/educare

CORREO: educare@una.cr

Meyer (2000) señala que la competencia intercultural es una combinación de habilidades sociales y comunicativas que incluyen la empatía, la resolución de conflictos, la capacidad para colaborar, la flexibilidad, el conocimiento de la lengua extranjera y el conocimiento de que la cultura; lleva consigo distintos estilos de discusión, velocidad del discurso, interpretación y patrones de pensamiento, técnicas para manejar las dificultades en la interacción, reflexión sobre el propio bagaje cultural y tolerancia a la ambigüedad. En general, el autor señala que la competencia intercultural implica la habilidad para integrar el propio bagaje cultural con otros. En este sentido, esta propuesta encaja con la enumeración de competencias sociales que propone Armstrong (2006), pues el autor señala que estas engloban: la influencia, la comunicación, el liderazgo, la apertura a los cambios, la resolución de conflictos, el establecimiento de relaciones, la colaboración y la cooperación, y las capacidades para trabajar en equipo.

Rodrigo (2003) expone que la comunicación intercultural puede entenderse como comunicación interpersonal en la que intervienen personas con referencias culturales suficientemente diferentes para crear barreras que podrían alterar la eficacia de la comunicación. En general, las distintas definiciones de la competencia intercultural coinciden en que se trata de la capacidad de ir más allá de la propia cultura y de interactuar con otras personas de diversos contextos tanto culturales como lingüísticos. En este sentido, el contacto con otras culturas y lenguas ofrece oportunidades para fomentar el desarrollo de la competencia comunicativa intercultural. Así, la cultura en el marco de la comunicación intercultural incluye los valores, los significados, los comportamientos, las costumbres y las creencias, entre otros; siguiendo a Witsel (2008, p. 14): "all the facets that determine the way of life of a group of people: their patterns of behaviour, and the ways in which they understand and interpret the world" [todas las facetas que determinan el modo de vida de un grupo de personas: sus patrones de comportamiento, y las formas en que entienden e interpretan el mundo].

Conocer una lengua extranjera y tener un buen nivel de esta no implica solo conocer bien las estructuras gramaticales sino que también lleva consigo comprender la cultura en que la lengua se halla inmersa y aprender el modo en que esa cultura se relaciona con otras, incluyendo la propia. Además, la lengua tiene un componente emocional y social (Beck et al., 2011) y si se trata de una lengua franca como es la lengua inglesa, se han de tener en cuenta aspectos de las distintas realidades sociales donde se emplea dicha lengua. Por lo tanto, es necesario que el alumnado que estudia una lengua extranjera, en este caso la lengua inglesa, desarrolle constantemente destrezas relacionadas con la comunicación intercultural, de manera que pueda participar en igualdad de condiciones en las distintas oportunidades que se le presenten para dialogar con personas de otras culturas.

Introducir las competencias interculturales es importante para promover la comunicación pacífica y el respeto a la diversidad (Baesler y Lauricella, 2014; Kruger, 2012). De hecho, estas competencias hacen que el alumnado desarrolle su creatividad al establecer relaciones entre 
la cultura propia y las otras, descubriendo nexos de unión que, a priori, no eran muy evidentes (Sainz, Soto y Almeida, 2011). Las competencias interculturales juegan un papel fundamental en la sociedad del conocimiento, debido a que permiten conocer las demandas y desafíos de las realidades socioculturales actuales, a la vez que ofrecen la posibilidad de profundizar en las distintas culturas (Priegue y Leiva, 2012).

Sin embargo, no es fácil trabajar con este tipo de competencias y tampoco son fáciles de evaluar (Byram, 2009; Byrnes, 2008; Schulz, Lalande, Dykstra-Pruim, Zimmer-Loew y James, 2005), tal y como señala Vinagre (2010, p. 299): "...la evaluación de la competencia intercultural es compleja y la investigación aún escasa". Este artículo pretende ser una contribución a la necesidad de introducir la competencia intercultural en el aula de lengua inglesa, al poner de manifiesto que las actividades llevadas a cabo en el aula pueden fomentar no solo el desarrollo de la competencia lingüística sino también intercultural.

\section{Contexto, participantes y metodología}

El alumnado que participa en este estudio cursaba la asignatura Lengua Inglesa V; asignatura obligatoria del Grado en Estudios Ingleses de la Universidad de Alicante. Se imparte en el tercer curso de grado y tiene seis créditos, es decir, hay 60 horas que se imparten en el aula, además de otras 90 horas en las que el alumnado ha de trabajar de manera individual o grupal fuera de clase para alcanzar las competencias establecidas en la asignatura.

Desarrollar las cinco destrezas (escucha, habla, lectura, escritura e interacción) a nivel C1 es el principal objetivo de la asignatura. Durante el curso académico 2014-2015 hubo 125 personas matriculadas en la asignatura, de las cuales 13 obtuvieron una beca Erasmus y estudiaron en otros países europeos. La mayoría del alumnado quiere trabajar como docente de inglés en el futuro o en empresas haciendo labores de traducción e interpretación.

La asignatura se imparte durante cuatro horas a la semana, una teórica y tres prácticas. La primera hora se centra en ofrecer al alumnado herramientas para mejorar la escritura académica: recursos cohesivos, la estructura del ensayo académico y las principales características de los distintos tipos de texto. En la segunda hora el alumnado expone una presentación oral grupal siguiendo los criterios presentados en clase. La siguiente hora se emplea para la revisión de ejercicios de gramática.

Cada clase de gramática comenzaba con una breve explicación teórica del tema gramatical objeto de estudio (el uso de artículos, el estilo indirecto, la voz pasiva, los adverbios, etc.); seguidamente se corregían ejercicios gramaticales de modo que el alumnado pudiera poner en práctica sus conocimientos gramaticales. Finalmente, la última hora consistía en la participación de un debate organizado por el grupo que había realizado la presentación oral en la clase anterior. 
doi: http://dx.doi.org/10.15359/ree.22-1.3

URL: http://www.una.ac.cr/educare

CORREO: educare@una.cr

Se optó por una metodología cooperativa debido al alto número de personas matriculadas en la asignatura. Por tanto, el alumnado se dividió en grupos de cinco o seis personas, hecho que llevaba consigo el desarrollo de competencias sociales como la cooperación, la escucha activa, la toma de decisiones, el reparto de tareas, el liderazgo, la capacidad para resumir y organizar la información, la comunicación, la resolución de conflictos, entre otras, además de la adquisición de contenidos. La profesora pidió a cada grupo que nombrara una persona coordinadora que sería responsable de coordinar el grupo y de interactuar con la profesora para consultar dudas y entregar las tareas requeridas. Al final del cuatrimestre tenía que entregar una evaluación del proceso de aprendizaje grupal prestando atención a lo aprendido y a los aspectos que mejorarían; en esta evaluación tenían que elegir las principales competencias adquiridas.

La profesora explicó al principio del cuatrimestre las distintas actividades cooperativas que se llevarían a cabo en la asignatura (ver sección 4). En este artículo nos vamos a centrar en presentar tres actividades. La metodología cooperativa que proponemos lleva consigo que el alumnado asume el protagonismo en el proceso de enseñanza-aprendizaje, mientras que el profesorado asume un papel facilitador o de guía en dicho proceso. Esta metodología también promueve la interacción como base para desarrollar las actividades propuestas de manera efectiva, de modo que se fomenta la adquisición de competencias sociales que serán fundamentales para la incorporación del alumnado en el mercado laboral.

Como parte de la metodología hemos de señalar que una vez que el alumnado había trabajado en el aula las distintas actividades cooperativas propuestas, la profesora diseñó una encuesta (Apéndice A) con el fin de conocer la opinión del alumnado sobre distintos aspectos relacionados con la competencia intercultural. Todo el alumnado que había participado en las actividades contestó la encuesta de manera anónima. Los datos se analizaron de manera manual.

\section{Propuesta de actividades cooperativas que promueven la interculturalidad}

Las actividades que presentamos a continuación fueron diseñadas con el objetivo de que el alumnado desarrollara su sensibilidad cultural y aumentara su interculturalidad, es decir, se trata de tareas que pretenden acercarlo a otras realidades culturales y que le invitan a reflexionar sobre las diferencias entre la propia cultura y las otras. Además, el hecho de trabajar en el aula con distintas realidades culturales y sociales le ayuda a ser más consciente de las diferencias y a fomentar actitudes de apertura y respeto a la diversidad. Por tanto, proponemos trabajar con temas globales (global issues) en el aula como las etnias, el género, etc., de modo que el alumnado amplíe su mirada y conozca aspectos relacionados con otras realidades culturales.

En consecuencia, el aprendizaje de la lengua inglesa que planteamos en Lengua Inglesa $\checkmark$ no solo consiste en el desarrollo de la competencia comunicativa prestando atención a aspectos lingüísticos, sociolingüísticos y pragmáticos, sino que además se ha de incorporar el componente intercultural, siguiendo las demandas de las sociedades del siglo XXI. 
Así, nuestra propuesta de actividades para la asignatura Lengua Inglesa $V$ no sigue las actividades que típicamente aparecen en las asignaturas de lengua inglesa, basadas en gramática estructural, en las que se va avanzando de estructuras más simples a más complejas a la vez que se va introduciendo vocabulario por campos semánticos progresivamente. Suelen ser programas basados en temas en los que se proponen trabajar las distintas destrezas.

\section{La presentación oral cooperativa}

El alumnado ha de realizar una presentación oral cooperativa sobre algún aspecto relacionado con la interculturalidad. De esta forma se le invita a reflexionar sobre posibles aspectos que se pueden tratar en una actividad de este tipo. La profesora explica las principales características de una presentación oral efectiva, empleando una presentación en Power Point y distintos videos donde se podían observar presentaciones con muchos aspectos a mejorar y otras con presentaciones bien organizadas.

Nos encontramos ante una actividad que fomenta la creatividad del alumnado, pues las indicaciones que la profesora dio al comienzo del cuatrimestre señalaban que cada miembro del grupo tenía unos siete minutos para la exposición, que se tenían que utilizar distintos recursos multimodales en esta y emplearse al menos diez referencias bibliográficas para su preparación, y que se tenían que tratar aspectos interculturales de modo que todo el alumnado pudiera profundizar en esta competencia.

El alumnado optó por realizar la presentación sobre distintos temas que incorporaban el componente intercultural a la docencia como, por ejemplo: a) aspectos culturales de distintos países en los que se habla la lengua inglesa, señalando las semejanzas y contrastando las diferencias, por ejemplo entre Inglaterra, Estados Unidos y Australia; b) aproximación a distintas religiones (budismo, cristianismo e islam) con el fin de observar las diferencias entre ellas y el efecto que las prácticas religiosas tienen en la vida de las personas; c) presentación de la gastronomía de distintos países y explicación de las razones por las que determinados alimentos se toman en algunas culturas y otros no.

\section{Aproximación a la gramática visual y al ensayo académico}

Con el fin de profundizar en la multimodalidad, la profesora enseñó al alumnado las principales pautas para leer imágenes siguiendo el modelo de gramática visual propuesto por Kress y van Leeuwen (2006). Se introdujeron los principales principios de composición (el valor de la información, el rasgo más sobresaliente y los marcos). La profesora señaló estos elementos en distintos textos de temática social y destacó la importancia de ver los distintos elementos que componen un texto multimodal como un todo, en el que la disposición de los distintos elementos tiene una finalidad comunicativa determinada. 
doi: http://dx.doi.org/10.15359/ree.22-1.3

URL: http://www.una.ac.cr/educare

CORREO: educare@una.cr

Seguidamente, con el fin de poder llevar a la práctica los aspectos presentados de forma teórica, la profesora seleccionó distintos textos de mujeres de diversas culturas de la publicación de la Organización No Gubernamental (ONG) Intermón Oxfam, IO; en concreto, se centró en las portadas. La clase se dividió en distintos grupos y a cada grupo se le dieron dos portadas, con el fin de que analizaran el modo en que las mujeres de distintas culturas aparecían representadas. El alumnado tenía que hacer un análisis siguiendo los principios de composición expuestos. Una vez hecho esto, en los grupos tenían que reflexionar acerca de la posible representación estereotipada de las mujeres en los textos (unidas a la pobreza, realizando labores domésticas, como madres, etc.) y cómo se las podría representar de otra forma con el fin de desterrar algunos estereotipos. A continuación, se pide al alumnado que comparta lo que sabe de los países y culturas a los que pertenecen las mujeres que aparecen en los textos. De esta forma, se pone en común en los grupos los conocimientos previos y se observa si hay imágenes estereotipadas sobre determinadas culturas.

Tras compartir en los pequeños grupos, se pidió que se nombrara un portavoz que pusiera en común las ideas debatidas. Una vez que se habían escuchado las ideas de todos los grupos, la profesora moderó un debate sobre el desconocimiento que se tiene de algunas culturas, en concreto de aspectos relacionados con la mujer, y cómo este lleva a que las personas perpetúen estereotipos que poco favorecen la integración y el respeto a la diversidad cultural.

Tras llevar a cabo esta actividad oral, la profesora explicó la estructura del ensayo académico mediante un video y una presentación en PowerPoint, después pidió al alumnado que escribiera un ensayo relacionado con los aspectos que se habían tratado de manera oral, de modo que pudieran expresar su opinión por escrito sobre aspectos relacionados con la interculturalidad por medio de un ensayo académico. Se invitó al alumnado a que reflexionara sobre las d entales y el modo en que se representan las mujeres de países del Sur. De esta forma, se partía de la perspectiva local y se ampliaba la perspectiva global, objetivo fundamental a la hora de conceder oportunidades al alumnado para que avance en la competencia intercultural.

\section{Aprendiendo vocabulario a través de textos de la prensa y de los libros de texto}

Nos parece fundamental que el alumnado trabaje con textos reales con el fin de que se establezca una relación directa entre lo que se enseña en las aulas universitarias y lo que ocurre en la sociedad. Por esta razón, la clase se dividió en seis grupos. A tres de ellos se les pidió que buscaran al menos cinco noticias en periódicos de habla inglesa en las que estuviera, de algún modo, presente el componente intercultural, bien de forma positiva o de forma negativa. Tras buscar las noticias, se les pidió que escribieran un breve resumen de las razones por las que habían seleccionado esas noticias. Antes de acabar esta actividad el alumnado tenía que preparar un glosario con, al menos, veinte términos relacionados con la interculturalidad. 
A los otros tres grupos se les pidió que consultaran en la biblioteca distintos libros de texto utilizados para enseñar inglés en distintos niveles, por ejemplo, B1, B2 y C1 y que prestaran atención a las distintas formas en las que el componente intercultural estaba presente en dichos libros. Al igual que los grupos anteriores, tras haber buscado los textos, tenían que escribir un resumen en el que se justificara la selección de textos llevada a cabo. Estos grupos también tenían que preparar un glosario.

Conceder libertad al alumnado para que seleccionara los textos favorece que se trabajen determinadas competencias como son el liderazgo y la toma de decisiones, ambas muy valoradas en el mercado laboral. También nos parece pertinente que la clase se divida en grupos y sean dos actividades distintas las que se realizan de modo que no todo el mundo esté trabajando con la misma actividad. En consecuencia, se concede dinamismo al proceso de enseñanza-aprendizaje y se permite al alumnado aprender no solo a través de la actividad que realiza, sino también a través de la actividad que realizan otros grupos.

\section{Discusión}

Las actividades presentadas en el apartado anterior ayudan a acercar al alumnado a realidades culturales diferentes de la propia por medio de textos reales o de temas relacionados con la competencia intercultural. En este sentido, se potencia que el alumnado tenga una visión global del mundo con el fin de que se enriquezca y pueda comprender mejor la realidad propia y la de las demás personas (Wang, Young, Wilhite y Marczyk, 2011). Se considera que las distintas culturas, experiencias y posicionamientos enriquecen de modo que las diferencias no se entienden como algo negativo. Además, trabajar con competencias interculturales ayuda a superar los modelos etnocéntricos en los que la sobrevaloración de los conocimientos y esquemas de la cultura mayoritaria conducen al rechazo o infravaloración de los conocimientos y esquemas de otras culturas.

Uno de los fines de las actividades propuestas es que el alumnado reflexione sobre distintas realidades, tome conciencia de distintos problemas y piense en posibles soluciones. Otro de los objetivos es que el alumnado aprecie y respete la diversidad cultural de modo que se promueva la integración de las personas culturalmente diferentes y se fomente la convivencia pacífica y la solidaridad para que se pueda enriquecer mientras aprende.

Conocer otras culturas y contrastarlas con la propia contribuye a acabar con el etnocentrismo y con actitudes que consideran la cultura propia como superior a otras de modo que se potencia el respeto a la diversidad y el hecho de que las diferencias culturales nos enriquecen. También se pretende que las actividades descritas ayuden al alumnado a desarrollar su habilidad para poder comprender e interactuar con otras culturas. Así, nos encontramos ante actividades que ayudan a romper estereotipos y prejuicios que limitan y hacen ver la realidad de una forma muy limitada. 
doi: http://dx.doi.org/10.15359/ree.22-1.3

URL: http://www.una.ac.cr/educare

CORREO: educare@una.cr

Las actividades que se han descrito en la sección anterior hacen que el alumnado reflexione sobre su propia realidad social y la contraste con otras de modo que las tareas contribuyan a que avance como ciudadanía global y al desarrollo de una visión mundialista de la realidad. Esto lleva consigo que participa en las actividades, crece como persona a la vez que contribuye al desarrollo colectivo. Además, se potencia la motivación, la curiosidad y la apertura para conocer realidades sociales diferentes de la propia y poder aprender de ellas, es decir, se integra la cultura propia con otras con el fin de adquirir la competencia intercultural al ver nexos de unión y diferencias entre distintas culturas.

Una idea fundamental que comparten las actividades propuestas es que no se enseña lengua y cultura per se, como materias separadas del contexto en el que ocurren, sino que se contextualizan las actividades para profundizar en las realidades sociales que presentan. En este sentido, se establecen relaciones entre los textos y el contexto en que se emplean, con el fin de que alumnado pueda ver en todo momento una aplicación práctica en las actividades que tiene que realizar.

Las actividades descritas en la sección anterior integran distintas destrezas (lectura, escritura, destreza oral, escucha e interacción) para que el alumnado pueda avanzar en todas las destrezas que hay que trabajar en el nivel requerido. Las actividades seleccionadas trabajan la lectura, ya que el alumnado tiene que leer sobre el tema social que elija para la presentación oral, la escritura al tener que redactar la presentación oral, la destreza oral por tener que hablar en clase sobre el tema elegido y sobre los temas que hay que debatir, la escucha al tener que escuchar las opiniones de las demás personas. También se potencia la interacción, al tener que participar en debates con el resto de sus compañeras y compañeros de clase, tener que discutir con el grupo el contenido de los textos consensuar el modo de organización del trabajo, entre otros. También se fomenta que el alumnado tome sus propias decisiones a la hora de seleccionar el tema de la presentación oral o los textos de prensa, de modo que se potencia la creatividad.

Nos encontramos ante actividades que incrementan distintas competencias sociales como son la comunicación, la escucha activa, el respeto a la diversidad de opiniones, entre otras. La comunicación es fundamental en una asignatura de lengua extranjera, de ahí que sean muchas las oportunidades para participar en la clase con el fin de que utilice la lengua inglesa en contextos tanto formales como informales con la ayuda de la profesora. El alumnado también tiene que seleccionar los aspectos de los temas propuestos que trata y el modo en que lo hace, escoge el vocabulario, la gramática y las estructuras gramaticales necesarias para que la comunicación sea efectiva.

En definitiva, se potencia la conciencia social con el fin de que el alumnado reflexione sobre la realidad de otras personas y pueda entender mejor las diferencias culturales y las desigualdades que se observan en el mundo, de modo que desarrolle la empatía y la comprensión. En consecuencia, el alumnado puede contrastar las situaciones sociales que se presentan en los temas y textos que se tratan en clase con su propia realidad, y desarrollar actitudes relacionadas con la solidaridad o el respeto a la diversidad. 
Además, en todo momento se fomenta la capacidad crítica, al incentivar la reflexión sobre situaciones sociales injustas y pensar en el modo en que se pueden mejorar, hecho que contribuye a que el alumnado asuma un compromiso activo en la transformación social. También se mejora la conciencia global y la toma de conciencia de problemáticas que pueden estar alejadas de la realidad personal, de modo que se abren nuevos horizontes y se desarrolla la conciencia social.

La encuesta que se diseñó con el fin de conocer la opinión del alumnado sobre determinados aspectos relacionados con la interculturalidad (ver apéndice A) encontró los siguientes resultados.

En la primera pregunta de la encuesta se preguntó al alumnado si consideraba necesario trabajar componentes interculturales en la formación universitaria en general y en su carrera en particular. El $78 \%$ ofreció una respuesta positiva frente al $22 \%$ que ofreció una respuesta negativa. En la segunda pregunta se le preguntó si se consideraba una persona intercultural. En esta ocasión, las tres cuartas partes del alumnado encuestado, en concreto, el $75.3 \%$ respondió que sí, frente al $24.7 \%$ que respondió que no. Según Byram y Fleming (1998, p. 8), las personas hablantes interculturales son aquellas que "establish a relationship between their own and the other cultures, to mediate and explain differences - and ultimately to accept that difference and see the common humanity beneath it" [establecen una relación entre la propia y otras culturas, para mediar y explicar las diferencias -y finalmente aceptar la diferencia y ver la humanidad común debajo de ella].

La pregunta tercera tenía como objetivo prioritario conocer si el alumnado tenía interés por conocer el modo de vida de otras personas y por presentar su propia cultura a otra gente. En este caso, el $84.2 \%$ de las respuestas son positivas frente al $15.8 \%$ que opinan lo contrario. La pregunta siguiente tiene como finalidad conocer si el alumnado considera que tiene habilidad para cambiar de perspectiva y para incorporar otros puntos de vista al propio. El 81.3\% considera que sí tiene esa habilidad frente al 18.75 que opina lo contrario.

En la pregunta cinco se pregunta al alumnado sobre qué competencias sociales de las ofrecidas en la lista le ayudan a ser más intercultural y las respuestas son variadas: el $42.4 \%$ opina que la empatía, un $38.8 \%$ considera que también la comunicación y considera que el resto de las competencias son adquiridas en menor medida: 9.9\% marca la cooperación, el 3.3\% la conciencia política, el $2.6 \%$ la gestión de conflictos, el $2 \%$ el liderazgo y solo el $1 \%$ marca la influencia (persuasión).

El hecho de que la empatía y la comunicación sean las dos competencias más votadas nos parece positivo, pues las actividades preparadas tenían como objetivo trabajar esas dos competencias fundamentalmente dentro de la clasificación de competencias sociales que proponen Tallon y Sikora (2011). Estas competencias son consideradas prioritarias, porque gracias a ellas el alumnado es capaz de conocer distintas realidades culturales y ponerse en el lugar de las personas que viven en estas, mostrar respeto por las demás culturas, aprender a comunicarse 
doi: http://dx.doi.org/10.15359/ree.22-1.3

URL: http://www.una.ac.cr/educare

CORREO: educare@una.cr

con los otros, y así lograr entender y aceptar las creencias ajenas, evitar prejuicios y el juzgar sin saber y sin conocer de primera mano distintas realidades culturales, eliminar prejuicios que vienen dados precisamente de la no interacción con personas de distintas culturas y costumbres. En definitiva, el hecho de que las actividades ofrezcan al alumnado oportunidades para ponerse en el lugar de las demás personas es esencial para entender otras culturas y formas de vida.

Las preguntas finales de la encuesta están basadas en Byram (2000), de ahí que en la pregunta 6 le preguntáramos al alumnado si le interesan las experiencias de la vida diaria de otras personas, en especial aquellos aspectos que no suelen presentarse a la gente de fuera a través de los medios de comunicación. El 78.9\% ofreció una respuesta afirmativa frente al $21.15 \%$ que respondió lo contrario.

La pregunta 7 consulta al alumnado sobre su interés en las experiencias diarias de una variedad de grupos sociales y no solo de la cultura dominante. El $80.6 \%$ respondió que sí, frente a un $19,4 \%$. El objetivo de la pregunta 8 es saber si el alumnado se daba cuenta de que es capaz de entender otras culturas cuando mira las cosas desde otro punto de vista y cuando mira su propia cultura desde otra perspectiva. El $85.8 \%$ respondió que sí, se puede observar que la mayoría considera que es capaz de entender otros puntos de vista.

La pregunta 9 consulta si el estudiantado conoce algunos hechos importantes sobre la vida de otra cultura y sobre el país, el Estado y la gente. Los resultados muestran que un $72.7 \%$ respondió que sí frente a un $27.3 \%$ que ofreció una respuesta contraria. En la pregunta 10 se preguntaba si sabe cómo iniciar una conversación con personas de la otra cultura y cómo mantenerla. Un $75.3 \%$ respondió que sí frente a un $24.7 \%$ que no.

Con la pregunta 11 se quiere saber si los estudiantes saben cómo resolver los malentendidos que surgen cuando la gente no es consciente del punto de vista de la otra cultura. Tres cuartas partes del alumnado encuestado, es decir, el $75 \%$ responde que sífrente al $25 \%$ que responden lo contrario. En la pregunta 12 se pregunta al alumnado si sabe cómo descubrir información nueva y aspectos nuevos de la otra cultura por sí mismo o misma. Un $82.9 \%$ responde que sí frente a un $17.1 \%$.

Tras el análisis de los resultados de la encuesta se puede observar que las respuestas positivas superan en gran medida a las negativas de modo que el alumnado expresa claramente su interés en otras culturas. Estos datos se relacionan con el hecho de que la mayoría ha tenido oportunidad de viajar a otros países, especialmente de habla inglesa, y ha tenido contacto con personas de otras culturas, de modo que ha podido experimentar dificultades o experiencias gratas relacionadas con encuentros interculturales.

Además, consideramosque habertrabajadodurantetodoel cuatrimestredistintas actividades que ayudaban a potenciar la interculturalidad enmarcadas en una metodología cooperativa ha contribuido a que el alumnado reflexione sobre la importancia de ser competente desde el punto 
de vista intercultural en general y como estudiantes del grado en estudios ingleses en particular. Como estudiantes de lenguas extranjeras, nos parece crucial que consideren importante que se introduzca el componente intercultural en el proceso de enseñanza-aprendizaje. Esto es relevante para el futuro pues una parte de estos grupos de estudiantes trabajarán fuera del país, de modo que estarán constantemente expuestos a otras culturas.

\section{Conclusiones}

Las actividades propuestas van más allá de la realidad local del alumnado, al introducir aspectos culturales a la vez que se aprende inglés. Así, se le ofrece una perspectiva más amplia de hacer y de ver las cosas con el fin de que se convierta en ciudadanía global, activa, crítica y responsable, capaz de participar activamente en las demandas de una sociedad cada vez más intercultural. En consecuencia, se potencia que aprenda a través del contexto social en que se desarrollan y en el que se presentan las actividades, de modo que se amplía la conciencia global gracias a los temas que se tratan y al intercambio comunicativo que tiene lugar en el proceso de enseñanza-aprendizaje. Además, potenciar las distintas destrezas implica que el alumnado aprende haciendo, mientras se fomenta la interacción en la clase, cuestiones esenciales en una pedagogía activa.

El hecho de que las distintas actividades propuestas sean cooperativas favorece el desarrollo de las destrezas interpersonales y la adquisición de competencias sociales en el alumnado, debido a que se trabaja el liderazgo, la escucha activa, el respeto a la diversidad de opiniones, entre otras. Por tanto, la propuesta presentada en este artículo va más allá de los contenidos que se han de enseñar siguiendo el currículo, sino que también se potencia la adquisición de competencias diversas, entre ellas la competencia intercultural. De hecho, trabajar la competencia intercultural implica el desarrollo de una conciencia global que lleve consigo desterrar algunos prejuicios y contribuir a que la sociedad sea más igualitaria.

Optarporuna metodología cooperativa favorecela participación delestudiantadoenelgrupo y la integración de las diferentes cualidades individuales y puntos de vista. Además, el aprendizaje cooperativo potencia las habilidades sociales y comunicativas, cuestiones fundamentales en la enseñanza de las lenguas extranjeras y en el fomento de intercambios interculturales. Nuestra propuesta educativa pretende ayudar al alumnado a avanzar como ciudadanía que apuesta por la igualdad de oportunidades y la solidaridad, de modo que se concede importancia a la interculturalidad y a la diversidad cultural para que se respeten las diferencias culturales y estas sean entendidas como un modo para enriquecernos y para favorecer el respeto a la diversidad. Por tanto, se ofrecen oportunidades para que el alumnado avance en su compromiso tanto con la realidad local como con la global y sea capaz de contribuir con la comunicación y con el intercambio entre culturas diferentes, al trabajar con textos que tratan sobre diversidad cultural y con actividades que potencian la reflexión sobre el componente intercultural. 
Si queremos que la competencia intercultural ocupe un lugar central en nuestras prácticas docentes, es necesario diseñar más actividades relacionadas con la convivencia con otras personas o con la cooperación entre personas de diferentes culturas, de manera que se ofrezcan, al alumnado, cuantas más posibilidades posibles para que pueda reflexionar sobre realidades sociales diferentes de la propia y así ampliar su mirada y adaptarse a la sociedad del siglo XXI, teniendo en cuenta que una de las características principales de esta es su dimensión global.

\section{Referencias}

Armstrong, M. (2006). A Handbook of human resource management practice (10 ed.). Londres: Kogan Page.

Baesler, E. J. y Lauricella, S. (2014). Teach peace: Assessing instruction of the nonviolent communication and peace course. Journal of Peace Education, 11(1), 46-63. doi: https:// doi.org/10.1080/17400201.2013.777899

Beck, L., Kumschick, I. R., Eid, M. y Klann-Delius, G. (2011). Relationship between language competence and emotional competence in middle childhood. Emotion, 12(3), 503-514. doi: https://doi.org/10.1037/a0026320

Bisquerra, R. (2008). Educación para la ciudadanía y la convivencia. El enfoque de la educación emocional. Madrid: Wolters Klumer.

Bisquerra, R. y Pérez, N. (2007). Las competencias emocionales. Educación XXI, 10, 61-82. doi: https://doi.org/10.5944/educxx1.1.10.297

Byram, M. (1997). Teaching and assessing intercultural communicative competence. Clavedon: Multilingual Matters.

Byram, M. (2000). Assessing intercultural competence in language teaching. Sprogforum, 18(6), 8-13. Recuperado de http://inet.dpb.dpu.dk/infodok/sprogforum/Espr18/byram.html

Byram, M. (2009). Evaluation and/or assessment of intercultural competence. En A. Hu y M. Byram, M. (Eds.), Intercultural competence and foreign language learning. Models, empiricism and assessment (pp. 215-234). Tübingen: Gunter Narr Verlag.

Byram, M. y Fleming, M. (Eds.). (1998). Language learning in intercultural perspective. Approaches through drama and ethnography. Cambridge: Cambridge University Press.

Byrnes, H. (2008). Articulating a foreign language sequence through content: A look at the culture standards. Language and Teaching, 41(1), 103-118. doi: https://doi.org/10.1017/ $\underline{\text { S0261444807004764 }}$ 
Clouet, R. (2013). Understanding and assessing intercultural competence in an online environment: a case study of transnational education programme delivery between college students in ULPGC, Spain, and ICES, France. RESLA, 26, 139-157.

Farh, C. I., Seo, M. G. y Tesluk, P. E. (2012). Emotional intelligence, teamwork effectiveness, and job performance: The moderating role of job context. The Journal of Applied Psychology, 97(4), 890-900. doi: https://doi.org/10.1037/a0027377

Francisco, A. (2010). Construyendo ciudadanía participativa en la sociedad de la información. Pixel Bit.Revista de Medios y Educación, 37, 135-146. Recuperado de http://www.redalyc. org/articulo.oa?id=36815118011

Johnson, D. W. y Johnson, F. P. (2009). Joining together: Group theory and group skills (10 ed.). Boston: Allyn and Bacon.

Johnson, D. W. y Johnson, R. T. (2009). Energizing learning: The instructional power of conflict. Educational Researcher, 38(1), 37-51. doi: https://doi.org/10.3102/0013189X08330540

Kress, G. y van Leeuwen, T. (2006). Reading images: The grammar of visual design ( $2^{\mathrm{a}} \mathrm{ed}$.). Londres: Routledge.

Kruger, F. (2012). The role of TESOL in educating for peace. Journal of Peace Education, 9(1), 17-30. doi: https://doi.org/10.1080/17400201.2011.623769

Lasnier, F. (2000). Réussier la formation par compétences. Montréal: Guérin.

Meyer, F. (2000). Wir waren, sind ein multinationales Haus: Zur Bedeutung und Aspekten interkultureller Kompetenz am Beispiel eines Dienstleistungsbetriebes. En Ressourcen, Kompetenzen, Qualifikationen: Potentiale von Zuwanderen in Weiterbildung und Arbeitsmarkt (pp. 17-26). Hamburg: Gesellschaft fur Arbeit, Technik, und Entwicklung.

Meyer, M. (1991). Developing transcultural competence: Case studies in advanced language learners. En D. Buttjes and M. Byram (Eds.), Mediating languages and cultures: Towards an intercultural theory of foreign language education (pp. 136-158). Clevedon: Multilingual Matters.

Priegue, D. y Leiva, J. J. (2012). Las competencias interculturales en la sociedad del conocimiento: Reflexiones y análisis pedagógico. EDUTEC. Revista Electrónica de Tecnología Educativa, 40, 1-12. Recuperado de http://www.edutec.es/revista/index.php/edutec-e/article/ view/370/107

Prieto, L. (2007). El aprendizaje cooperativo. Madrid: PPC.

Rodrigo, M. (2003). Comunicación intercultural. Barcelona: Anthropos. 
doi: http://dx.doi.org/10.15359/ree.22-1.3

URL: http://www.una.ac.cr/educare

CORREO: educare@una.cr

Sainz, M., Soto, G. y Almeida, L. (2011). Competencias socio-emocionales y creatividad según el nivel de inteligencia. Revista Electrónica Interuniversitaria de Formación del Profesorado, 14(3), 97-106. Recuperado de http://www.redalyc.org/articulo.oa?id=217022109008

Schulz, R. A., Lalande, J. F., Dykstra-Pruim, P., Zimmer-Loew, H. y James, C. J. (2005). In pursuit of cultural competence in the german language classroom: Recommendations of the AATG task force on the teaching of culture. Die Unterrichtspraxis / Teaching German, 38(2), 172181. doi: https://doi.org/10.1111/j.1756-1221.2005.tb00055.x

Tallon, R. y Sikora, M. (2011). Conciencia en acción. Eneagrama, inteligencia emocional y cambio (Trad. de N. Steinbrun). Madrid: Alquimia.

Vinagre, M. (2010). El aprendizaje intercultural en entornos virtuales de colaboración. RESLA, 23, 297-317.

Vinagre, M. (2014). El desarrollo de la competencia intercultural en los intercambios telecolaborativos. RED, Revista de Educación a Distancia, 13(41), 1-22.

Wang, N., Young, T. Wilhite, S. C. y Marczyk, G. (2011). Assessing students' emotional competence in Higher Education: Development and validation of the widener emotional learnihg scale. Journal of Psychoeducational Assessment, 29(1), 47-62. doi: 1 https://doi. org/10.1177/0734282909359394

Witsel, M. (2008). Transcultural teachers: Experiences of academics teaching tourism and hospitality in multicultural and transnational contexts (Tesis doctoral). Southern Cross, Lismore, NSW. Recuperado de http://epubs.scu.edu.au/cgi/viewcontent. cgi?article $=1082 \&$ context $=$ theses 


\section{Apéndice $A$}

Encuesta sobre la competencia interculturalidad

1. ¿Crees que es necesario trabajar componentes interculturales en la formación universitaria en general y en tu carrera en particular?

2. ¿Te consideras una persona intercultural?

3. ¿Tienes interés por conocer el modo de vida de otras personas y por presentar tu propia cultura a otras personas?

4. ¿Tienes habilidad para cambiar de perspectiva y para incorporar otros puntos de vista al tuyo?

5. ¿Cuáles de las siguientes competencias sociales (Tallon y Sikora, 2011) te pueden ayudar a ser más intercultural?

\begin{tabular}{|l|l|}
\hline Empatía & \\
\hline Conciencia política & \\
\hline Comunicación & \\
\hline Cooperación & \\
\hline Liderazgo & \\
\hline Influencia (persuasión) & \\
\hline Gestión de conflictos & \\
\hline \hline
\end{tabular}

61. ¿Te interesan las experiencias de la vida diaria de otras personas, en especial aquellos aspectos que no suelen presentarse a los de fuera a través de los medios de comunicación?

7. ¿Estás interesada/o en las experiencias diarias de una variedad de grupos sociales dentro de una sociedad y no solo de la cultura dominante?

8. ¿Te das cuenta de que eres capaz de entender otras culturas cuando miras las cosas desde otro punto de vista y cuando miras tu propia cultura desde la perspectiva de otros/as?

9. ¿Conoces algunos hechos importantes sobre la vida de la otra cultura y sobre el país, el Estado y la gente?

10. ¿Sabes cómo iniciar una conversación con personas de la otra cultura y cómo mantenerla?

11. ¿Sabes cómo resolver los malentendidos que surgen cuando la gente no es consciente del punto de vista de la otra cultura?

12. ¿Sabes cómo descubrir información nueva y aspectos nuevos de la otra cultura por ti misma/o?

${ }^{1}$ Las preguntas 6 a 12 están basadas en Byram (2000, p. 4). 